\title{
Amphotericin B induced transient encephalopathy
}

\author{
M Somathilaka $^{1}$, D C Peiris ${ }^{2}$, J Wanigasinghe ${ }^{3}$ \\ Sri Lanka Journal of Child Health, 2013; 42(1): 48-49
}

(Key words: Amphotericin B; transient encephalopathy)

\section{Introduction}

Systemic and renal toxicity of Amphotericin B $(\mathrm{AmB})$ is well established ${ }^{1}$. However, its neurotoxic effects are less frequently reported. Those reported include leucoencephalopathy ${ }^{2}$, drowsiness and parkinsonism-like features ${ }^{3}$. We report a case of $\mathrm{AmB}$ induced transient encephalopathy in a child with rhabdomyosarcoma.

\section{Case report}

A fifteen month old boy with a rhabdomyosarcoma of the upper lip was treated with chemotherapy using a combination of Ifosfamide, vincristine, actinomycin-D and doxorubicin. This resulted in an excellent response with rapid reduction of tumour mass. During the third course of chemotherapy he developed a marked febrile neutropenia. This was complicated with gram negative septicaemia causing typhilitis (neutropenic enterocolitis). The child had no neurological symptoms related to this septicaemia. This was treated with broad spectrum antibiotics. Concomitant $\mathrm{AmB}$ was added to treat the extensive oral candidiasis. Although the fever and symptoms related to typhilitis resolved, on the third day of AmB therapy the child became unusually irritable with altered behaviour and later became unusually drowsy. These neurological symptoms progressively worsened to an encephalopathic state over the next three days. There was loss of voluntary movements with poor head control and he did not appear to fix or follow. There was no response to command. Speech was limited to an intermittent irritable cry.

\section{${ }^{1}$ Senior Registrar in Paediatric Oncology, \\ ${ }^{2}$ Consultant Paediatric Oncologist, National Cancer Institute Maharagama, ${ }^{3}$ Senior Lecturer and Honorary Consultant Paediatric Neurologist, University of Colombo}

(Received on 27 April 2012: Accepted after revision on 25 May 2012)
By this time, apart from a thrombocytopenia (28,000/cu mm), all other cell counts had returned to normal. All markers of infection, liver and renal functions were normal. Blood sugar, electrolytes, serum calcium, magnesium and potassium were normal. Computerised tomography showed no evidence of cerebral oedema, haemorrhage or central nervous system (CNS) infection. The sleep EEG showed subtle attenuation of sleep background activity. Lumbar puncture was deferred due to persistent thrombocytopenia.

He was continued on the broad spectrum antibiotics together with acyclovir, but showed no response over first 72 hours of encephalopathy. Serum electrolytes and sugars were carefully maintained within normal ranges. In the absence of any haematological or radiological evidence of underlying systemic fungal infection a decision was taken to withhold AmB therapy. Within the first 12 hours of this, we noted a dramatic improvement in his behaviour, recognition of his mother, reappearance of speech and regain of his motor functions. Within 24 hours he was seated on his mother's lap being able to request for his food. Within two days of discontinuing $\mathrm{AmB}$ he was back to his normal pre-illness state.

The sudden unexplained onset of encephalopathy and dramatic response after discontinuation of AmB therapy hinted at AmB induced encephalopathy. However, our inability to perform lumbar puncture (due to thrombocytopenia) deprived us of the opportunity of ruling out the remote possibility of CNS infection as a cause. He had no signs of CNS infection apart from encephalopathy and the acyclovir commenced at the beginning did not result in any improvement. Discontinuation of acyclovir as soon as the child started improving did not result in any deterioration either. Therefore, the sequence of events and dramatic response to withdrawal of therapy 
supported a clinical diagnosis of transient encephalopathy due to $\mathrm{AmB}$ in this child.

\section{Discussion}

Chemotherapy induced encephalopathy is not uncommon in oncological practice. Ifosfamide, nitrosoureas, cyclophosphamide and methotrexate are agents known to cause encephalopathy ${ }^{4}$. However, chemotherapy related encephalopathy requires treatment with the offending agent over a longer duration and at higher doses. Chemotherapy also can result in hepatic and renal encephalopathies, hyponatraemia or syndrome of inappropriate antidiuretic hormone (SIADH) secretion particularly following vincristine and Ifosfamide administration ${ }^{5}$. In our patient another cause of this encephalopathy could have been metastatic spread to the CNS, which is common with this tumour type especially when the rhabdomyosarcoma has parameningeal involvement. However, the neuroimaging excluded this possibility. Moreover metastatic spread is extremely unlikely in those with excellent response to initial chemotherapy.

AmB, a polyene antifungal drug, although commonly giving rise to early renal and hepatic toxicity, only rarely gives rise to neurotoxicity ${ }^{1}$. Neurotoxic effects reported include a leucoencephalopathy due to the myelin breakdown ${ }^{2}$. The mechanism of this white matter damage is poorly understood. However, it may be related to the AmB induced pore formation in the host cell membrane, where the sterols are found. Sterols are the primary membrane target for the action of $\mathrm{AmB}^{6,7}$. This $\mathrm{AmB}$ induced white matter changes on magnetic resonance imaging has reported to resolve, once the treatment is discontinued. Other reported CNS effects of AmB include transient encephalopathy like in our patient $^{1,2}$, secondary parkinsonism in children undergoing allogenic bone marrow transplantation and treated with high-dose amphotericin B and progressive neurologic dysfunction (dementia, akinesia, mutism, hyperreflexia, and tremor $)^{3}$.

In our child, the diagnosis of transient AmB induced encephalopathy was based on clinical hypothesis due to our inability to perform lumbar puncture. However, awareness of this entity helped us to avoid unnecessary investigations and prolongation of empirical therapies.

\section{References}

1. Laniado-Laborin $\mathrm{R}$, Cabrales-Vargas $\mathrm{MN}$. Amphotericin B: side effects and toxicity. Revista Iberoamericana de Micologia 2009; 26(4): 223-7. http://dx.doi.org/10.1016/j.riam.2009.06.003

2. Ellis WG, Sobel RA, Nielsen SL. Leukoencephalopathy in patients treated with amphotericin B methyl ester. Journal of Infectious Diseases 1982; 146(2): 125-37. http://dx.doi.org/10.1093/infdis/146.2.125

3. Mott SH, et al. Encephalopathy with parkinsonian features in children following bone marrow transplantations and high-dose amphotericin B. Annals of Neurology 1995; 37(6): 810-4.

http://dx.doi.org/10.1002/ana.410370616

4. Filley CM, Kleinschmidt-DeMasters BK. Toxic leukoencephalopathy. New England Journal of Medicine 2001; 345(6): 425-32. http://dx.doi.org/10.1056/NEJM200108093450 $\underline{606}$

5. Robertson GL, Bhoopalam N, Zelkowitz L.J. Vincristine neurotoxicity and abnormal secretion of antidiuretic hormone. Archives of Internal Medicine 1973; 132(5): 717-20. http://dx.doi.org/10.1001/archinte.1973.03650 110061013

6. Baginski M, Czub J. Amphotericin B and its new derivatives: mode of action. Current Drug Metabolism 2009; 10(5): 459-69. http://dx.doi.org/10.2174/13892000978889801 $\underline{9}$

7. Balmaceda CM, et al. Reversal of amphotericin B related encephalopathy. Neurology 1994; 44(6): 1183-4. http://dx.doi.org/10.1212/WNL.44.6.1183 